\title{
Extramedullary Hematopoiesis Presenting as a Right Adrenal Mass in a Patient With Beta Thalassemia
}

\author{
Hossein Karami ${ }^{1}$; Mehrnoush Kosaryan ${ }^{2}$; Mehrdad Taghipour ${ }^{3, *}$; Rayka Sharifian ${ }^{2}$; Aili \\ Aliasgharian ${ }^{1} ;$ Mohsen Motalebi $^{3}$ \\ ${ }_{1}^{1}$ Thalassemia Research Center, Mazandaran University of Medical Sciences, Sari, IR Iran \\ 2 Mazandaran University of Medical Sciences, Sari, IR Iran \\ ${ }^{3}$ Nephrology and Urology Research Center, Baqiyatallah University of Medical Sciences, Tehran, IR Iran \\ ${ }^{*}$ Corresponding author: Mehrdad Taghipour, Nephrology and Urology Research Center, Baqiyatallah University of Medical Sciences, Tehran, IR Iran. Tel: +98-2181262073, Fax: +98- \\ 2181262073, E-mail: mehrdadtaghipour@gmail.com
}

Received: April 13, 2014; Accepted: June 14, 2014

\begin{abstract}
Introduction: Extramedullary hematopoiesis in the kidney and adrenal are rarely reported in medical literature and are usually found as incidentaloma. It usually occurs in patients with hematologic disorder such as thalassemia.

Case Presentation: The patient was a 23-year-old Iranian man with beta thalassemia who was admitted with a suprarenal mass. Adrenal mass was detected by ultrasonography and computed tomography. Results of biochemical evaluations were insignificant. The patient underwent right adrenalectomy. Gross and microscopic histopathologic examination demonstrated extramedullary hematopoiesis without any adrenal tissue.

Conclusions: To the best of our knowledge, we document the first reported case of adrenal extramedullary hematopoiesis in Iran, which seems to be rare and remarkable.
\end{abstract}

Keywords:Adrenal; Hematopoiesis; Thalassemia

\section{Introduction}

Beta thalassemia, caused by a decrease in production of $\beta$-globin chains, affects multiple organs and is associated with considerable morbidity and mortality (1). Extramedullary hematopoiesis (EMH) is a normal compensatory reaction to insufficient bone marrow function to maintain the circulatory demands. It is seen in thalassemia, myelofibrosis, polycythemia, leukemia, lymphoma, after bone marrow irradiation, poisoning, and neoplastic conditions (2-4). EMS arises in organs that are evolved from pluripotential stem cells (5). Although EMS of the kidney has been reported in medical literature, it is a rare pediatric adrenal mass, which is clinically referred to as incidentaloma. These cases often occur in patients with hematologic disorders one of which is thalassemia. To the best of our knowledge, we documented the first reported case of adrenal EMH in Iran.

\section{Case Presentation}

The patient was a 23-year-old Iranian man with beta thalassemia who was admitted with a suprarenal mass. Adrenal mass was detected by ultrasonography (US) and computed tomography (CT). Liver size was normal and without a bulky mass in US. A heterogeneous mass was observed in the superior part of the right kidney with scattered echogenic areas, suggesting small scattered calcification and internal cystic areas. Spleen size was 150 $\mathrm{mm}$ without mass. Contrast-enhanced spiral CT images showed $10 \times 12 \mathrm{~cm}$ bulky mass in the right kidney region, containing necrotic and calcified areas. The borders of the lesion were clear and regular and no edema was seen in surrounding fat (Figure 1). Liver and spleen were enlarged but adenopathy and ascites were not observed. Results of biochemical evaluations were insignificant. The patient underwent right adrenalectomy. The resected mass weighed about $370 \mathrm{~g}$. In the histopathologic study, the tissue was brown fragile and homogenous. Gross and microscopic examination demonstrated EMH. In histopathologic examination of the liver, stage I, grade II chronic hepatitis and hemochromatosis were reported. Hepatic sample was tested for hepatitis $C$ virus genotypes $1 \mathrm{a}, 1 \mathrm{~b}, 2$, and $3 \mathrm{a}$ that yielded nontypable genotype; the reason could be low number of virus copy in the serum or presence of genotypes (subtypes) other than tested ones. The results of hepatitis $C$ virus RNA loading test was negative. The complications of the patient's disease were under control. 

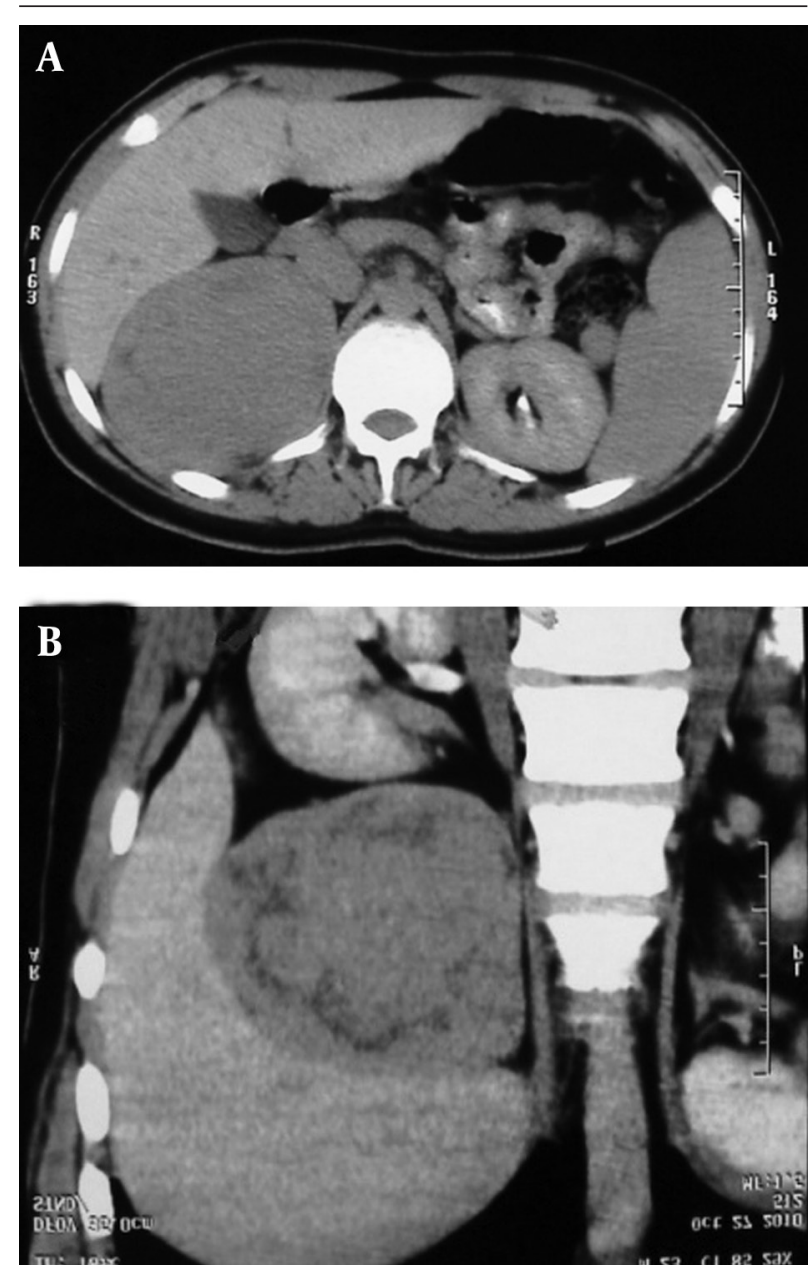

Figure 1. A Bulky Mass in the Region of Right Kidney, Containing Necrotic and Calcified Areas, Is Visible in Above Computed Tomography Images

\section{Discussion}

EMH is a physiologic compensatory event in many hematologic diseases $(6,7)$. It occurs most commonly in the spleen, liver, and lymph nodes and less frequently in the lung, pleura, breast, thymus, small bowel, and central nervous system. EMH is rarely found in the kidneys as well as the adrenal glands $(5,6)$. These sites are normally involved in active hematopoiesis in the fetus during gestation. There are two forms of EMH, namely, para-osseous and extra-osseous. In former, the normal medullary tissue of the bone marrow ruptures through the bone to present as a para-osseous mass and in latter, EMS occurs within soft tissue (8). The incidence of the EMH in patients with polytransfused major thalassemia is reported to be less than $1 \%(6)$.

There are few reports on EMH in the adrenal gland in patients with thalassemia. In an Italian case, the authors reported a rare case of adrenal EMH in a 10-year-old Caucasian female with beta thalassemia. The lesion was clinically discovered as incidentaloma of the right adrenal gland and was resected. Histopathologic examination of the mass demonstrated normal adrenal parenchyma surrounding EMH, which contained hematopoietic cells at various stages of maturation with predominance of the erythroid cell lines. The authors reported the female to male ratio of 2:1 and the mean age of 35 years (range, 10-66). Its prevalence was reported as $3.6 \%$ of all surgically removed adrenal incidentaloma (5). According to histopathologic findings, when EMH is associated with agnogenic myeloid metaplasia, the prevalence would be about 5\% to 6\% (5). Adrenal EMH might be detected as an incidentaloma as well as a histopathologic finding (5). Adrenal gland might be involved unilaterally (similar to our case) or bilaterally (5).

Chuang et al. reported a case of adrenal EMH in a 27-year-old woman with beta thalassemia. She underwent cholecystectomy for gallstones and splenectomy for splenomegaly at the age of 20. Then she was admitted to hospital due to a palpable nontender mass in the right subcostal area. US, CT, and magnetic resonance imaging disclosed a right suprarenal mass of $7.5 \times 5.8 \mathrm{~cm}$ size. Surgical exploration and biopsy confirmed the diagnosis of EMH tumor. The tumor was left in situ. Subsequent follow-up showed no increase in the tumor size and no progression of anemia. The patient remained asymptomatic and no additional EMH tumor developed. Surgery or radiotherapy should be performed only when EMH tumors cause symptoms such as spinal cord compression. To avoid unnecessary surgical procedures, adrenal EMH tumors should be considered in patients with thalassemia and an adrenal mass (9).

Major thalassemia is a widespread genetic anemia in Mediterranean countries as well as north and west of Africa $(10,11)$. Beta thalassemia major is the most common autosomal disorder in Iran, particularly in Mazandaran Province $(12,13)$; nevertheless, after a comprehensive research for the literature concerning this subject, we found no reports of adrenal EMH among Iranian patients with thalassemia. To the best of our knowledge, we documented the first patient of adrenal EMH in Iran, which seems to be rare and remarkable. Adrenal EMH might be clinically detected as incidentaloma (5). Adrenal incidentaloma in association with hematologic disorders, e.g. agnogenic myeloid aplasia or beta thalassemia, needs careful imaging as well as adrenal hormonal investigation in order to exclude malignancy and subclinical hypersecretory syndromes. US-or CT-guided FNA of the lesion is useful in diagnosis of EMH (5). Although EMH is a rare cause of adrenal mass, the diagnosis must be considered in any patient with a history of a congenital hemolytic disorder. In this regard, undergoing unnecessary procedures and their associated morbidities might be avoided. Regular transfusion therapy might prevent EMH by correcting anemia and thereby terminating the stimulus for EMH. Surgical decompression is the management method of choice because the histopathologic diagnosis can be established and immediate decompres- 
sion of the mass can be achieved. The disadvantages of the surgical intervention include risk of excessive bleeding due to the high vascularity of the mass and high incidence of recurrence (8).

\section{Acknowledgements}

The authors gratefully thank the members of Thalassemia Research Center staff (Bu-Ali-Sina Hospital SariIran) for their help in reporting this case.

\section{Authors' Contributions}

Hossein Karami: Clinician; Mehrnoush Kosaryan: Clinician; Mehrdad Taghipour: Data collection, writing and editing of the manuscript Rayka Sharifian: Data collection Aili Aliasgharian: editing of the manuscript.

\section{References}

1. Rund D, Rachmilewitz E. Beta-thalassemia. $N$ Engl J Med. 2005;353(11):1135-46.

2. Saghafi M, Shirdel A, Lari SM. Extramedullary hematopoiesis with spinal cord compression in beta-thalassemia intermedia. EurJ Intern Med. 2005;16(8):596-7.

3. Campisi S, Mangiagli A, De Sanctis V, Giovannini M. Paraplegia in a thalassaemic patient with short stature. Pediatr Endocrinol Rev. 2011;8 Suppl 2:340-4.

4. Alam MR, Habib MS, Dhakal GP, Khan MR, Rahim MA, Chowdhury AJ, et al. Extramedullary hematopoiesis and paraplegia in a patient with hemoglobin e-Beta thalassemia. Mymensingh Med J. 2010;19(3):452-7.

5. Porcaro AB, Novella G, Antoniolli SZ, Martignoni G, Brunelli M, Curti P. Adrenal extramedullary hematopoiesis: report on a pediatric case and update of the literature. Int Urol Nephrol. 2001;33(4):601-3.

6. Haidar R, Mhaidli H, Taher AT. Paraspinal extramedullary hematopoiesis in patients with thalassemia intermedia. Eur Spine J. 2010;19(6):871-8.

7. Calhoun SK, Murphy RC, Shariati N, Jacir N, Bergman K. Extramedullary hematopoiesis in a child with hereditary spherocytosis: an uncommon cause of an adrenal mass. Pediatr Radiol. 2001;31(12):879-81.

8. Habibzadeh F, Yadollahie M, Haghshenas M. Extra-medullary haematopoiesis in beta-thalassaemia. J Postgrad Med. 2005;51(1):45-6.

9. Chuang CK, Chu SH, Fang JT, Wu JH. Adrenal extramedullary hematopoietic tumor in a patient with beta-thalassemia. J Formos Med Assoc. 1998;97(6):431-3.

10. Rashidi Ghader F, Kosaryan M, Karami H. Mazandaran Evaluation of treatment effect of Deferiperone \& Desferrioxamine on cardiac complication in major thalassemic patients. Medic Sci J. 2008;17(61):12-20.

11. Karami H, Kosaryan M, Abolghasemi H, Rashidi Ghader F, Vahidshahi K, Dabiryan.. Comparison of therapeutic effect of Deferiperone \& Desferrioxamine with Desferrioxamine in major thalassemic patients. Blood. 2009;7(4):227-34.

12. Kosaryan M, Mahdavi MR, Valaee N, Rahbar F, Moradi. . Effect of Ferros sulfat prescription on hemoglobin A2 level Mazandaran. Medic Sci J. 2002;12(35):53-60.

13. Khorasani G, Kosaryan M, Vahidshahi K, Shakeri S, Nasehi MM. Results of the national program for prevention of beta-thalassemia major in the Iranian Province of Mazandaran. Hemoglobin. 2008;32(3):263-71. 